as demonstrated in the Understanding Potential Long-Term Improvements in Function with Tiotropium (UPLIFT $®$ ) trial [4]. The authors are correct in highlighting the notable differences between the Lung Health Study and UPLIFT ${ }_{\circledR}$ study populations with respect to age and disease severity. It is of interest, however, that, in the Lung Health Study, a post hoc subanalysis of the annual rates of decline by smoking category (continuing smokers, intermittent smokers and sustained quitters), baseline lung function (range 90-55\% predicted FEV1) and age (range 35-70 yrs) showed that baseline disease severity (FEV1\% predicted) was not predictive of the annual change in FEV1 in $\mathrm{mL} \cdot \mathrm{yr}^{-1}$ within any smoking category, whereas older subjects who were either continuing or intermittent smokers had a slightly more rapid annual decline in lung function than younger subjects in the same smoking categories, although among quitters age did not influence FEV1 decline [2]. These findings suggested that smoking cessation leads to generally comparable benefits (in terms of slowing disease progression) across the spectrum of middle to older age and mild to moderate disease severity. The UPLIFT ${ }_{\circledast}$ trial results appear to confirm and extend the findings from the Lung Health Study to an older and more severely diseased COPD population.

J. Zielinski and co-workers suggest that the UPLIFT ${ }_{\circledast}$ data be formally analysed further to compare the rates of decline among the three smoking categories. Unfortunately, such a subgroup analysis would be problematic, since the three subgroups are defined by post-randomisation smoking status and are not comparable with respect to such baseline variables as age, Global Initial for Chronic Obstructive Lung Disease stage, FEV1 \% predicted and sex [4].
D.P. Tashkin*, B. Celli", S. Kesten ${ }^{\star}$, T. Lystig ", S. Mehra ${ }^{+}$and M. Decramer ${ }^{\S}$

*David Geffen School of Medicine at UCLA, Los Angeles, CA, "Brigham and Women's Hospital, Boston, MA, 'BoehringerIngelheim Pharmaceuticals, Ridgefield, CT, ${ }^{+}$Pfizer Pharmaceuticals, New York, NY, USA, and ${ }^{\S}$ University of Leuven, Leuven, Belgium.

Correspondence: D.P. Tashkin, Dept of Medicine, David Geffen School of Medicine at UCLA, 10833 Le Conte Ave, Los Angeles, CA, USA. E-mail: dtashkin@mednet.ucla.edu

Statement of Interest: Statements of interest for all authors can be found at www.erj.ersjournals.com $/ \mathrm{misc} /$ statements.dtl

\section{REFERENCES}

1 Anthonisen NR, Connett JE, Kiler JP, et al. Effects of smoking intervention and the use of an anticholinergic bronchod.lator on the rate of deline in FEV1. The Lung Health Study. JAMA 1994; 272: 1497-1505.

2 Scanlon PD, Connett JE, Waller L, et al. Smoking cessation and lung function in mild-to-moderate chronic obstructive pulmonary disease: The Lung Health Study. Am J Respir Crit Care Med 2000; 161: 381-390.

3 Anthonisen NR, Connett JE, Murray RP. Smoking and lung function of Lung Health Study participants after 11 years. Am J Respir Crit Care Med 2002; 166: 675-679.

4 Tashkin DP, Celli B, Kesten S, et al. Long-term efficacy of tiotropium in relation to smoking status in the UPLIFT trial. Eur Respir J 2009; 103: 963-974.

DOI: $10.1183 / 09031936.00036110$

\title{
Polyacrylate nanoparticles: toxicity or new nanomedicine?
}

\section{To the Editors:}

Nanotoxicology is now becoming a very important field in view of the unique physicochemical properties of nanomaterials that may lead to adverse biological effects on occupational, environmental and consumer matters. However, a conclusion on the toxicity of nanomaterials should be carefully drawn taking into account the complexity of determining the toxicity of nanoscale materials. Until now, studies on the adverse outcomes of nanomaterials, such as carbon nanotubes and nanoparticles, were limited to the experimental stage. Although there were some reports on human cell lines, the research was limited to in vitro studies; in vivo studies were completely limited to animals. Very recently, researchers from Beijing Chaoyang Hospital, Beijing, China, have reported the first case of the clinical toxicity of polyacrylate nanoparticles in humans [1]. The main evidence involves pathological examination of patients' lung tissue and observations by transmission electron microscopy. Nonspecific pulmonary inflammation, pulmonary fibrosis and foreign-body granulomas of pleura were displayed. Round nanoparticles $\sim 30 \mathrm{~nm}$ in diameter were observed to lodge in the cytoplasm and karyoplasm of pulmonary epithelial and mesothelial cells and also in chest fluid. Based on these clinical observations and analysis, the authors conclude that long-term exposure to polyacrylate nanoparticles could result in serious damage to human lungs. Here, we note that their work may provide important evidence for concerns about the toxicity of nanoparticles in humans and may afford promising opportunities to develop a new clinical therapeutic treatment for such nanoparticle-based diseases. However, we would like to point out that there are not enough data to strongly suggest that the clinical symptoms of the patients result from the toxicity of 
polyacrylate nanoparticles themselves. Therefore, adequate experimental evidence is very necessary for supporting the claimed toxicity, such as in vivo and in vitro toxicological assessments of polyacrylate nanoparticles, control experiments using animals in the laboratory that simulate work conditions, toxicological experiments of other chemicals in the paste, etc.

The first concern is the existence of polyacrylate nanoparticles. We argue that it is not known what nanoparticles (chemistry and composition) are found in patients (see question 3 in SONG et al. [1]). The authors agreed that polyacrylate has often been regarded as low toxicity, but some nanoparticles, such as silicon nanoparticles, thin zinc oxide, titanium dioxide, nanoscale silver clusters, are normally added into the polyacrylate emulsion to make the material stronger and more abrasion resistant [1]. The authors claimed that they have more evidence to show that the nanoparticles contained in the polyacrylate emulsion had possibly caused the disease. What is this evidence? No related data were presented. Could it be said that the evidence is in the literature cited? We actually do not know whether some nanoparticles are added into the paste in this clinical case. More surprisingly, the authors finally reached a conclusion that all data presented in the study suggested that the polyacrylate nanoparticles are linked to the patients' illnesses. Indirect analysis is really not enough.

The authors claimed that using electron microscopy, nanoparticles ( $\sim 30 \mathrm{~nm}$ in diameter) are found in both the paste and the dust particles. As an extremely simple measure, an energy dispersive spectrometer analysis may be used to characterise the chemical composition of the nanoparticles mentioned. However, in the study, the authors did not present any analysis relating to the chemical composition of the nanoparticles. We suggest that the nanoparticles may be ascribed to the long-term accumulation of organic molecules. Besides, the size of nanopaticles always follows the normal distribution. Are polyacrylate nanoparticles, dust particles or any other nanoparticles contained in the polyacrylate emulsion? We admit to being confused. It is therefore reasonable to doubt that there are polyacrylate nanoparticles in the paste.

To support their claim about the toxicity of polyacrylate nanoparticles, SonG et al. cite two studies $[2,3]$. We have read these studies with interest, but unfortunately have to say that they do not support their claim. One evaluates the acute toxicity of oral exposure to nanoscale zinc powder in mice [2], the other investigates the effectiveness of various models for blood particle attachment that are applicable to complex flow fields with relatively high particle counts [3]. There is no information about polyacrylate nanoparticles.

Assuming that there really are polyacrylate nanoparticles in the paste, the authors ignored the possibility of a secondary effect of adsorption by polyacrylate nanoparticles. It has been demonstrated that carbon nanotube toxicity may be mistakenly attributed to direct toxicity, when in fact it is a secondary effect of adsorption of essential micronutrients from cell culture medium $[4,5]$. Here, we consider that the conditions in real workplaces are more complicated than that of experiments under laboratory conditions. It is difficult to imagine that only pure polyacrylate nanoparticles have direct contact with human lungs without any other chemicals. The authors claimed that, although the toxicity of the polyacrylate nanoparticles in patients has not been firmly established, animal studies and in vitro experiments may provide clues relating to the nanoparticles themselves. We do not know why the authors cited other different materials such as nano- and micron-sized particles of cobalt-chromium alloy [6], singlewalled carbon nanotubes [7] and carbon nanoparticles [8] to support the possibility of the toxicity of polyacrylate nanoparticles. We argue that citing the literature does not expand to include this possibility. We cannot simply deduce $A \rightarrow C$ from $A \rightarrow B$ and $B \rightarrow C$. What is more, the authors suggested that the patients' illness appears to be a "nanomaterial-related disease" because the compounds in the paste are low in toxicity and are unlikely to cause disease of the severity seen in these patients. Are essential micronutrients in cell culture medium toxic? The answer absolutely is no, but more experiments have demonstrated that adsorption of essential micronutrients from cell culture medium onto a single-walled carbon nanotube could result in some damage, as mentioned earlier. And, in fact, there have been some reports on the toxicity of toluene [9-11]. Significantly, the in vitro effects of straight-chain alkanes (nC6nC10), and benzene and toluene on pulmonary alveolar macrophages and lysosomal enzymes in rat and rabbit lungs was studied in 1987 [12].

If it is true that organ molecules adsorbed by polyacrylate nanoparticles have an adverse effect on human lungs, the work by SoNG et al. [1] may be the best example for drug delivery in humans and provide opportunities for further advances in nanomedicine. One of the most important applications, nanoparticle-based drug delivery is currently attracting much attention from the medical field because nanoparticles have the potential to pass across physiological barriers or target specific cells and organs and administer small quantities of drugs [13, 14]. Now scientists have developed methods for loading a variety of medicine onto polymeric nanoparticles to form new nanomedicine [15-25]. In particular, E. Turos and co-workers (University of South Florida, Tampa, FL, USA), have found that polyacrylate nanoparticles can act as carriers for drug delivery [26-30]. New nanomedicine contains several types of antibiotic-conjugated polyacrylate nanoparticles and shows activity against methicillin-resistant Staphylococcus aureus and Bacillus anthracis and no cytotoxicity towards human dermal cells. This team also conducted an in vivo experiment of penicillin-conjugated nanoparticle emulsion in determining toxicological responses initiated upon systemic and topical application in a murine model [31]. Favourable results suggest that polyacrylate nanoparticle-containing emulsions may afford promising opportunities for treating both skin and systemic infections. Edward Turos is also co-inventor of a US patent application (Pub. No.: WO/2009/055650) by the University of South Florida the polyacrylate nanoparticle drug delivery for treatment of anthrax and malaria

Other important evidence showing the biocompatibility of polyacrylate is the work by K. Aoshiba and co-workers (Tokyo Women's Medical University, Tokyo, Japan) [32]. The group investigated the therapeutic effects of polyacrylate-stabilised platinum nanoparticles (PAA-Pt) on pulmonary inflammation in smoking mice. Intranasal administration of PAA-Pt prior to cigarette smoke (CS) exposure was found to inhibit depletion of antioxidant capacity, nuclear factor- $\kappa \mathrm{B}$ activation and 
neutrophilic inflammation in the lungs of mice. Another important result of this work is that intranasal administration of PAA-Pt alone did not elicit pulmonary inflammation or toxicity. In in vitro experiments, treatment of alveolar-type-IIlike A549 cells with PAA-Pt inhibited cell death after exposure to a CS extract.

Obviously, polyacrylate nanoparticles hold great potential to be developed as drug carriers for nanomedicine and there will be further applications for various diseases in the future. Indeed, the majority of work is still in the early stages of development, but given that this field is only a few years old, we believe it is only time before the proposed drug delivery will ultimately impact disease research and human clinical trials.

Not only the Chinese government and scientists but also scientists worldwide have paid close attention to this first nanomaterial-related accident in humans. On September 1-3, 2009, over 1,500 scientists from over 40 countries and regions gathered at Beijing International Convention Center to attend the China International Conference on Nanoscience and Technology (ChinaNano 2009, Beijing, China). During the 3day meeting, one of the major issues of nanotechnology of nanoscale materials discussed by scientists worldwide was to encircle the work by SoNG et al. [1]. One of the themes was that scientists from European and American countries should form a survey group to investigate this first clinical case. The purpose of this response is not to disagree with the potential risk of nanoparticles, but to make clear that rigorous and systemic tests must be used before a conclusion can be reliably reached. If not, it might lead to the positive effects of nanoscience and nanotechnology for the development of society as a whole being ignored, and cause a psychological panic in people. There could also be consequences for future governmental policy guidance on nanoscience and nanotechnology.

Again, numerous studies have attempted to address the toxicity issues associated with the administration routes of different nanomaterials, but no formal evaluation guidelines and few definite trends have been established. The work of SONG et al. [1] presents a cautionary tale about the clinical toxicity of nanomaterials in humans; undoubtedly, the timing is very good. We take this opportunity to make an appeal to the nanotechnology community to establish a safety assessment database of nanomaterials, including the properties of nanomaterials (composition, size, architecture, etc.), standardisation of experiments (especially, physiological conditions), toxicological assessment methods and thorough comparison across various animal models, etc., for a comprehensive evaluation. As in the case of the potential biological/clinical effects of nanomaterials in the pharmaceutical industry, the availability of formal evaluation guidelines could be of enormous benefit.

\section{H. Ren* and X. Huang",}

*National Center for Nanoscience and Technology of China, Beijing, "The Key Laboratory of Biomimetic Sensing and Advanced Robot Technology, Anhui Province, Institute of Intelligent Machines, Chinese Academy of Sciences, Hefei, China. "Dept of Biochemistry, Oxford University, South Parks Road, Oxford, UK.
Correspondence: X. Huang, The Key Laboratory of Biomimetic Sensing and Advanced Robot Technology, Anhui Province, Institute of Intelligent Machines, Chinese Academy of Sciences, Hefei 230031, China. E-mail: xhbioch@hotmail.com

\section{SUPPORT STATEMENT}

This work was supported by the One Hundred Person Project of the Chinese Academy of Sciences, China, and the National Natural Science Foundation of China (Grant No. 90923033) to X. Huang. H. Ren is supported by the 973 Key Project (Grant No. 2006CB932504).

Statement of Interest: None declared.

\section{REFERENCES}

1 Song Y, Li X, Du X. Exposure to nanoparticles is related to pleural effusion, pulmonary fibrosis and granuloma. Eur Respir J 2009; 34: 559-567.

2 Wang B, Feng WY, Wang TC, et al. Acute toxicity of nano- and micro-scale zinc powder in healthy adult mice. Toxicol Lett 2006; 161: 115-123.

3 Longest PW, Kleinstreuer C. Comparison of blood particle deposition models for non-parallel flow domains. J Biomech 2003; 36: $421-430$.

4 Guo L, Bussche AVD, Buechner M, et al. Adsorption of essential micronutrients by carbon nanotubes and the implications for nanotoxicity testing. Small 2008; 4: 721-727.

5 Casey A, Herzog E, Lyng FM, et al. Single walled carbon nanotubes induce indirect cytotoxicity by medium depletion in A549 lung cells. Toxicol Lett 2008; 179: 78-84.

6 Papageorgiou I, Brown C, Schins R, et al. The effect of nano- and micron-sized particles of cobalt-chromium alloy on human fibroblasts in vitro. Biomaterials 2007; 28: 2946-2958.

7 Shvedova AA, Kisin ER, Mereer R, et al. Unusual inflammatory and fibrogenic pulmonary responses to single-walled carbon nanotubes in mice. Am J Physiol Lung Cell Mol Physiol 2005; 289: L698-L708.

8 Hamilton RF, Buford MC, Wood MB, et al. Engineered carbon nanoparticles alter macrophage immune function and initiate airway hyper-responsiveness in the BALB/c mouse model. Nanotoxicology 2007; 1: 104-117.

9 Bushnell PJ, Oshiro WM, Samsam TE, et al. A dosimetric analysis of the acute behavioral effects of inhaled toluene in rats. Toxicol Sci 2007; 99: 181-189.

10 Thrall KD, Woodstock AD. Evaluation of the dermal absorption of aqueous toluene in F344 rats using real-time breath analysis and physiologically based pharmacokinetic modeling. J Toxicol Environ Health 2002; 65: 2087-2100.

11 Thrall KD, Weitz KK, Woodstock AD. Use of real-time breath analysis and physiologically based pharmacokinetic modeling to evaluate dermal absorption of aqueous toluene in human volunteers. Toxicol Sci 2002; 68: 280-287.

12 Suleiman SA. Petroleum hydrocarbon toxicity in vitro: effect of $\mathrm{n}$ alkanes, benzene and toluene on pulmonary alveolar macrophages and lysosomal enzymes of the lung. Arch toxicol 1987; 59: 402-407.

13 Sung JC, Pulliam BL, Edwards DA. Nanoparticles for drug delivery to the lungs. Trends Biotechnol 2007; 25: 563-570.

14 Otsuka H, Nagasaki Y, Kataoka K. PEGylated nanoparticles for biological and pharmaceutical applications. Adv Drug Deliv Rev 2003; 55: 403-419.

15 Dailey LA, Jekel N, Fink L, et al. Investigation of the proinflammatory potential of biodegradable nanoparticle drug delivery systems in the lung. Toxicol Appl Pharmacol 2006; 215: 100-108. 
16 Dailey LA, Kleemann E, Wittmar M, et al. Surfactant-free, biodegradable nanoparticles for aerosol therapy based on the branched polyesters, DEAPA-PVAL-g-PLGA. Pharm Res 2003; 20: 2011-2020.

17 Grenha A, Seijo B, Remunan-Lopez C. Microencapsulated chitosan nanoparticles for lung protein delivery. Eur J Pharm Sci 2005; 25: 427-437.

18 Shi LJ, Plumley CJ, Berkland C. Biodegradable nanoparticle flocculates for dry powder aerosol formulation. Langmuir 2007; 23: 10897-10901.

19 Zhang Q, Shen ZC, Nagai T. Prolonged hypoglycemic effect of insulin-loaded polybutylcyanoacrylate nanoparticles after pulmonary administration to normal rats. Int J Pharm 2001; 218: 75-80.

20 Pandey R, Sharma A, Zahoor A, et al. Poly (DL-lactide-co-glycolide) nanoparticle-based inhalable sustained drug delivery system for experimental tuberculosis. J Antimicrob Chemother 2003; 52: 981-986.

21 Bivas-Benita $\mathrm{M}$, Romeijn S, Junginger $\mathrm{HE}$, et al. PLGA-PEI nanoparticles for gene delivery to pulmonary epithelium. Eur J Pharm Biopharms 2004; 58: 1-6.

22 Gou ML, Zheng L, Peng XY, et al. Poly(epsilon-caprolactone)poly(ethylene glycol) -poly(epsilon-caprolactone) (PCL-PEG-PCL) nanoparticles for honokiol delivery in vitro. Int J Pharm 2009; 375: 170-176.

23 Khemtong C, Kessinger CW, Gao JM. Polymeric nanomedicine for cancer MR imaging and drug delivery. Chem Commun 2009; 3497-3510.
24 Patil YB, Toti US, Khdair A, et al. Single-step surface functionalization of polymeric nanoparticles for targeted drug delivery. Biomaterials 2009; 30: 859-866.

25 Beck-Broichsitter M, Gauss J, Packhaeuser CB, et al. Pulmonary drug delivery with aerosolizable nanoparticles in an ex vivo lung model. Int J Pharm 2009; 367: 169-178.

26 Abeylath SC, Turos E. Glycosylated polyacrylate nanoparticles by emulsion polymerization. Carbohydr Polym 2007; 70: 32-37.

27 Abeylath SC, Turos E, Dickey S, et al. Glyconanobiotics: Novel carbohydrated nanoparticle antibiotics for MRSA and Bacillus anthracis. Bioorg Med Chem 2008; 16: 2412-2418.

28 Turos E, Shim JY, Wang Y, et al. Antibiotic-conjugated polyacrylate nanoparticles: New opportunities for development of anti-MRSA agents. Bioorg Med Chem Lett 2007; 17: 53-56.

29 Turos E, Reddy GSK, Greenhalgh K, et al. Penicillin-bound polyacrylate nanoparticles: Restoring the activity of beta-lactam antibiotics against MRSA. Bioorg Med Chem Lett 2007; 17: 3468-3472.

30 Garay-Jimenez JC, Young A, Gergeres D, et al. Methods for purifying and detoxifying sodium dodecyl sulfate-stabilized polyacrylate nanoparticles. Nanomedicine 2008; 4: 98-105.

31 Greenhalgh K, Turos E. In vivo studies of polyacrylate nanoparticle emulsions for topical and systemic applications. Nanomedicine 2009; 5: 46-54.

32 Onizawa S, Aoshiba K, Kajita M, et al. Platinum nanoparticle antioxidants inhibit pulmonary inflammation in mice exposed to cigarette smoke. Pulm Pharmacol Ther 2009; 22: 340-349.

DOI: $10.1183 / 09031936.00022410$

\section{Asthma predictive factors in infants with bronchiolitis: asthma risk at 13-20 years of age}

\section{To the Editors:}

MATRICARDi et al. [1] presented an interesting comparison on the persistence of wheezing at 10-13 yrs of age between two birth cohorts from the UK and Germany. When the cohorts were re-evaluated by harmonising the data, family history of asthma, absence of recurrent rhinitis at $<1 \mathrm{yr}$, recurrent chest infections at $<2$ yrs and atopic sensitisation at $<3-4$ yrs were independent risk factors for wheezing at 10 years, with some differences between early life wheezers and non-wheezers [1]. When three or four of these risk factors were present, the algorithm was highly specific but not sensitive in predicting persistent wheezing at $>10 \mathrm{yrs}$ of age: sensitivity 0.53 , specificity 0.85 and positive likelihood ratio 6.38 [1].

CASTRO-Rodriquez et al. [2] introduced the first asthma predictive index (API) based on the Tucson birth cohort study to predict asthma risk at school age in children who wheezed at $<36$ months of age. Major risk factors were asthma in parents and atopic dermatitis in the child, and minor risk factors were allergic rhinitis, wheezing apart from cold and blood eosinophilia. Loose index (wheezing at $<36$ months and one major or two minor criteria) means moderate asthma risk, and stringent index (repeated wheezing at $<36$ months and one major or two minor criteria) means high asthma risk. The $85 \%$ specificity and $42 \%$ sensitivity of the loose index mean a positive likelihood ratio of 2.2 with a modest predictive impact.
The $97 \%$ specificity and $16 \%$ sensitivity of the stringent index mean a likelihood ratio of 1.2 only.

API was later modified (mAPI) by adding atopic sensitisation to inhaled allergens, confirmed by allergen-specific immunoglobulin E measurements or positive skin tests, as a minor criterion [3]. Though this modification was an obvious improvement with a more objective risk assessment, mAPI has not been validated by follow-up studies.

The results of long-term Scandinavian post-bronchiolitis follow-ups have highlighted the need to revise the algorithms to be more suitable for infants treated for bronchiolitis in hospital, as summarised in two reviews $[4,5]$. The outcome of early life wheezers is connected with disease severity, invasiveness of infection and triggering virus not possible to be taken into account in cohort studies with parent-reported symptoms. In addition, treatment in hospital and outpatient clinics of hospitals enables assessment of more objective risk factors by virological and allergological examinations. The suggested hospitalised children's API (hAPI) consists of three major criteria (parental asthma, atopic dermatitis or food allergy in the child and sensitisation to inhaled allergens) and three minor criteria (sensitisation to food allergens, wheezing by viruses other than RSV and blood eosinophilia when healthy) [1, 2]. In addition, parental smoking, maternal smoking in particular, further increases the asthma risk. 